\title{
Organization of Grid Resources in Communities
}

\author{
Asif Akram \\ e-Science Centre, CCLRC Daresbury \\ Laboratory \\ Warrington, UK, WA4 4AD \\ +44 1925603790 \\ a.akram@dl.ac.uk
}

\author{
Rob Allan \\ e-Science Centre, CCLRC Daresbury \\ Laboratory \\ Warrington, UK, WA4 4AD \\ +44 1925603207 \\ r.j.allan@dl.ac.uk
}

\begin{abstract}
Locating suitable resources within a Grid is a computationally intensive process, with no guarantee of quality and suitability of the discovered resources. An alternative approach is to categorize resources based on the services they provide - leading to the interaction of peers with common goals to form societies/communities. The communities can be adaptive in nature and evolve based on changes in their operating environment -such as changes in neighboring communities and user requirements.
\end{abstract}

\section{INTRODUCTION}

Emerging distributed computing paradigms, such as Grid Computing, comprise of dynamic and distributed resources/peers. A middleware is required which can organize these resources as a "Virtual Organizations". Resource discovery is a time-consuming process and imposes an overhead on network access in Grid Systems. The numbers of interactions are likely to increase exponentially as the numbers of peers grow. Restricting interactions between set of peers is a key factor to scale the resource discovery problem. This helps us to define two terms, Expertise and Interest [4], [5]. Expertise of a peer is the basic service provided by that peer and Interest of a peer is the service/services provided by other peers which are supportive to its main service. A similar problem in Grid Computing is what Davis and Smith refer to as the "connection problem" [1], where peers need to find other suitable peers to co-operate with, assist, or interact with. "Focused Addressing" [2] is one solution to the connection problem where requests are sent to particular subset of peers, believed to assist the requesting peer.

\section{COMMUNITY FORMATION}

A middleware is required to assist a new peer in the Grid to discover the Service Peer which may have interest in its capabilities/services. If the interests of a Service Peer are different, the new peer is either referred to any suitable Service Peer/s, or the new peer queries the middleware to locate alternative Service Peer/s with compatible interests. A Service

Permission to make digital or hard copies of all or part of this work for personal or classroom use is granted without fee provided that copies are not made or distributed for profit or commercial advantage and that copies bear this notice and the full citation on the first page. To copy otherwise, or republish, to post on servers or to redistribute to lists, requires prior specific permission and/or a fee.

MGC 2006, November 27, 2006, Melbourne, Australia.

Copyright 2005 ACM 1-58113-000-0/00/0004...\$5.00.
Peer and all peers registered with it constitute a community. A Service Peer manages all peers within the community and communicates with neighboring communities on the behalf of member peers. A middleware is essential for the bootstrapping of a new peer, as it supports a new peer to discover enough network resources to sustain itself. We therefore also foresee the existence of common infrastructure services (such as monitoring, directory, security/certificate authority, etc) within each community.

\section{TYPE OF COMMUNITIES}

Individual autonomous peers have expertise and interests in specific resource/s. Based on these expertise and interests, peers are grouped together, but expertise and interests are not the only criteria for categorizing peers. Communities/societies can be of different types as mentioned below:

Competing Community: In a Competing Community each peer has the same expertise - although some service attributes may vary. Similarity in services may develop competition amongst member peers, as members compete each other to get selected.

Co-Operative Community: In Co-Operative communities peers provide different services, which must be used alongside services of other members. Hence, when any peer is selected, then the possibility of selection of another member peer providing utility service/s increases. This mutual co-operation is suitable for peers which provide simple services.

Goal Oriented Community: This is collection of peers work together to achieve a particular goal. Goal oriented communities are important in self-organizing systems, where interactions between member peers are not pre-defined, but the services required are. In such instances, member peers may interact with each other in arbitrary ways to achieve a given end result.

Ad Hoc Community: In ad hoc communities peers interact directly with each other without interference and involvement of a Service Peer. Peers belonging to different communities providing supporting services form the basis of an ad hoc community.

Domain-Oriented Community: Such a community is formed by linking together similar-minded organizations and institutions, instead of the services they provide, such as academic communities, research communities, and open-source communities. Hence these communities are domain-oriented rather than service-oriented.

Virtual Community: The Virtual community is a community of communities. This effect is achieved by leasing out the member Peer to other community for certain time period, before that lease period either Service Peer requests to renew the lease of corresponding Peer or it can't use the service of the Peer directly. 
Sharing Community: In this type of community different Communities share their resources with each other; this sharing of resources is not restricted to member Peers but includes core and optional services. Community A may have QoS monitoring module, which it shares with Community B assuming either Community B doesn't have such module or Community A may has more advanced monitoring module.

\section{PROTOTYPE and RESULTS}

For simulation purposes the JXTA prototype has been developed with option for creating Groups and Peers along with their description. This description is used as the one of the membership criteria, when any Peer applies for the membership. The community is created along with randomly generated External Rating and community assigns randomly generated Internal Rating to all of its members at the time of membership. Peers apply for membership based on high external rating of the community. The community grants membership based on overall rating of the peer and description of the peer.

Prototype was evaluated with different set of parameters i.e. maximum number of member Peers in a community, maximum number of communities joined by single peer etc. Evaluation results were quite encouraging, and similar pattern was observed by changing the set of parameters. In the beginning of the evaluation following four steps were quite frequent:

- Selection of communities by peers

- Compatibility checks i.e. rating and description by peers.

- Request for membership by peers

- Membership confirmation from the community.

The preliminary result with different set of constraints is shown below in the tabular and graphical form:

Table 1. Membership Acceptance-Request Ratio

\begin{tabular}{|c|c|c|c|}
\hline \multirow{2}{*}{$\begin{array}{c}\text { Groups } \\
\text { Discovered }\end{array}$} & \multicolumn{3}{|c|}{ Membership Acceptance/Request Ratio } \\
\cline { 2 - 4 } & $\begin{array}{c}\text { Group Size } \\
(50)\end{array}$ & $\begin{array}{c}\text { Group Size } \\
(80)\end{array}$ & $\begin{array}{c}\text { Group Size } \\
(100)\end{array}$ \\
\hline $0-100$ & $80 / 100=0.8$ & $100 / 100=1.0$ & $100 / 100=1.0$ \\
\hline $100-200$ & $60 / 80=0.75$ & $76 / 90=0.85$ & $90 / 100=0.9$ \\
\hline $200-300$ & $30 / 50=0.6$ & $50 / 75=0.66$ & $63 / 85=0.74$ \\
\hline $300-400$ & $10 / 25=0.4$ & $28 / 50=0.45$ & $28 / 55=0.5$ \\
\hline $400-500$ & $0 / 5=0$ & $3 / 18=0.16$ & $5 / 21=0.23$ \\
\hline
\end{tabular}

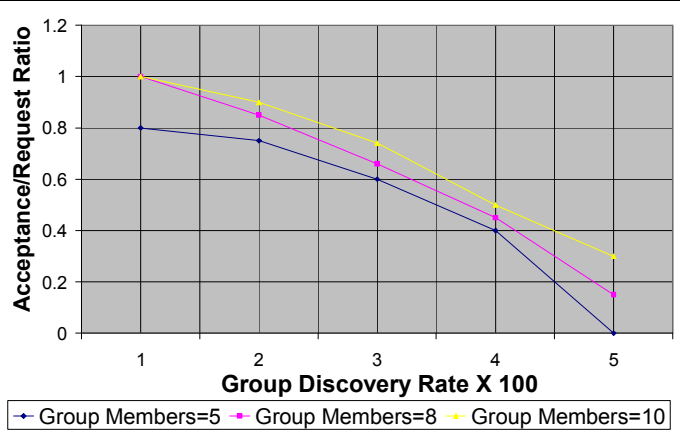

Figure 1. Membership Acceptance-Request Ratio vs. Peer Group Discovery Rate
In the table above the left column 'Groups Discovered' compares with the ratio of total membership requests and the accepted requests. It is obvious from the above table initially peer applies for most of discovered communities but this trend decreases when more communities are discovered. With the passage of time frequency of request for membership decreases and the rate of membership granted by communities decreases because either all member peers have better rating or their expertise matches the interest of community. Once System becomes stable then even peers don't apply for membership or changes group.

The time required by the environment to achieve stable state depends on the constraints set by system i.e. rate of community discovery, number of member peers in a single community and number of communities joined by single peer. We are confidant that organizing resources into different communities will give new dimension to Grid Computing.

\section{CONCLUSION AND SUMMARY}

In this paper, we have presented the concept of categorizing peers in communities on the basis of their expertise and interests. Organizing peers in one form or another makes the discovery of resources efficient, whilst minimizing computational overheads. Categorizing the peers in communities is simple, open and easy to implement, and the initial overhead of developing communities pays-off latter at the time of resource discovery. Communities are more stable, and stability increases with the passage of time, communities have a simple learning time and are more adaptive to operate in a dynamic environment. We have proposed the external and internal rating for communities and peers respectively which may be used to support a given Quality of Service, effective participation of autonomous peers and better interaction among communities and member peers. A JXTA implementation of a prototype system is discussed to describe the salient features of our approach. A key theme of this work is to determine how communities should be structured to support resource discovery, and how particular roles within a community can be used to determine interactions between participants within a community, and those between participants across community. This work extends techniques and results discussed in [3].

\section{REFERENCES}

[1] Davis, R. and R. G. Smith, Negotiation as a Metaphor fro Distributed Problem Solving. Artificial Intelligence 20, 63109, 1983.

[2] Parunak, H. V. D., Distributed Artificial Intelligence, Chapt. Manufacturing Experionce With the Contract Net, pp. 285310, Research Notes in Artificial Intelligence. Los Altos, CA: Morgan Kaufmann Publishers, 1987.

[3] Steven Lynden and Omer Rana, Coordinated Learning to support Resource Management in Computational Grids Second International Conference on Peer-to-Peer Computing, (2002) 81-89

[4] Leonard Foner. Yenta: A multi-agent, referral-based matchmaking system. In Proceedings of the 1st International Conference on Autonomous Agents, (1997) 301-307.

[5] Bin Yu and Munindar P. Singh: Searching Social Networks. Proceedings of Second International Joint Conference on Autonomous Agents and Multi-Agent Systems, 2003. 
